\title{
Time for Change: Why Learning Analytics Needs Temporal Analysis
}

\author{
Simon Knight \\ Faculty of Transdisciplinary Innovation \\ University of Technology Sydney \\ Simon.Knight@uts.edu.au
}

\author{
Alyssa Friend Wise \\ Steinhardt School of Culture, Education, and Human Development \\ New York University \\ alyssa.wise@nyu.edu \\ Bodong Chen \\ College of Education and Human Development \\ University of Minnesota \\ chenbd@umn.edu
}

\section{IMPORTANCE AND CHALLENGES FOR TEMPORALITY IN LEARNING ANALYTICS}

Learning is a process that occurs over time: We build understanding, change perspectives, and develop skills over the course of extended experiences. As a field, learning analytics aims to generate understanding of, and support for, such processes of learning. Indeed, a core characteristic of learning analytics is the generation of high-resolution temporal data about various types of actions. Thus, we might expect study of the temporal nature of learning to be central in learning analytics research and applications. However, temporality has typically been underexplored in both basic and applied learning research. As Reimann (2009) notes, although "researchers have privileged access to process data, the theoretical constructs and methods employed in research practice frequently neglect to make full use of information relating to time and order" (p. 239). Typical approaches to analysis often aggregate across data due to a collection of conceptual, methodological, and operational challenges. As described below, insightful temporal analysis requires (1) conceptualising the temporal nature of learning constructs, (2) translating these theoretical propositions into specific methodological approaches for the capture and analysis of temporal data, and (3) practical methods for capturing temporal data features and using analyses to impact learning contexts. There is a pressing need to address these challenges if we are to realize the exciting possibilities for temporal learning analytics. 
(2017). Time for change: Why Learning analytics needs temporal analysis. Journal of Learning Analytics, 4(3), 7-17. http://dx.doi.org/10.18608/jla.2017.43.2

\section{$1.1 \quad$ Conceptual Challenges}

Time is implicit in many theories of learning as the general period over which change occurs. However, the details of how processes are expected to unfold over time are rarely well conceptualised. As a result, temporal constructs are underspecified and those that do exist are generally quite simple (Littleton, 1999; Mercer, 2008; Mercer \& Littleton, 2007; Barbera, Gros, \& Kirschner, 2014). For example, the construct of distributed practice is temporal in that it refers to events over time, indicating that learning occurs better when the same amount of practice (or studying) is spaced out over time than if it is compressed into a short period. But temporal concerns are not specified beyond basic assertions about the duration of the time gap between study sessions (see Cepeda, Pashler, Vul, Wixted, \& Rohrer, 2006). Robust research on temporality requires thoughtful conceptualisation (and operationalisation) of constructs salient to learning tasks with respect to temporality. Theoretically grounded constructs can then be mapped to expected patterns in trace data that analyses can confirm (or refute).

To do this, the field of learning analytics requires substantial conceptual development. At a foundational level, a clear conceptual understanding of "temporality" and its importance for learning is required. In one sense, temporality refers to the passage of elapsed time and comes with a collection of related concepts such as duration, rate, and acceleration (e.g. Blikstein, 2011; Haythornthwaite \& Gruzd, 2012). In other cases, however, the term temporality is used to refer to the order of events without explicit reference to the duration of passage of time between them (e.g. Halatchliyski, Hecking, Goehnert, \& Hoppe, 2014; Biswas, Jeong, Kinnebrew, Sulcer, \& Roscoe, 2010). Thus, we recognize that in education studying sequences of change and progression can provide insight that is complementary to research that addresses the measured flow and passage of time. These two types of "temporal" analyses are quite distinct in the claims that they can support, thus it is important to be clear which kind of temporal consideration and analysis is being attempted in any particular study.

Toward this end, conceptual work is needed to specify learning constructs and their temporal components. In many cases, theories presume that different activities take place in some order or in some flow over time, but exact sequences, durations, and gaps are not detailed. For example, Wise and Chiu (2011) conducted a temporal analysis of the process of knowledge construction among groups of students. Despite the existence of a five phase theoretical model proceeding from "sharing information" to "agreeing and applying" (Gunawardena, Lowe, \& Anderson, 1997), a detailed description of the process of moving through these phases did not exist. For example: Do group members always move through the phases in sync with each other? Is it possible to return to earlier phases or skip phases entirely? A clear conceptual account of the different progressions possible was required to specify how the data should be modeled in this work. This example illustrates how a move to engage in temporal analyses requires us to be more specific in our conceptual propositions. 
(2017). Time for change: Why Learning analytics needs temporal analysis. Journal of Learning Analytics, 4(3), 7-17. http://dx.doi.org/10.18608/jla.2017.43.2

\section{$1.2 \quad$ Methodological Challenges}

A separate set of challenges for temporal analyses are methodological in nature. The increased availability of digital-trace and video data has led to an increase in the quantity and types of time-based data available. However, the temporal information associated with the data is not always well used (Kapur, Voiklis, \& Kinzer, 2008; Wise, Perera, Hsiao, Speer, \& Marbouti, 2012), which may limit the explanatory power of these analyses (Akhras \& Self, 2000; Reimann, 2009). Analyses following traditional quantitative approaches have tended to focus on comparing and correlating relatively aggregated variables (such as the percentage of discourse turns that demonstrate metacognition). Such aggregation fails to fully utilize the temporal information embedded in the data (Kapur, Voiklis, \& Kinzer, 2008), reducing the power of the analysis. Such information reduction may also limit the validity of the conclusions drawn (Reimann, 2009). Thus, we need to employ richer analysis approaches to fully exploit the temporal nature of our data.

A move to do so raises new important methodological questions. Two critical questions relate to the construction of appropriate time windows and analytic time units (Wise, in preparation). The time window is the overall period over which learning activities are analysed. This may be the whole time for which data was collected, or some conceptually (or, at times empirically) justified smaller segment of it. For example, data logs from a classroom chat tool might be collected over the course of a year but analysed in weekly windows defined by the topic. The time window is thus a tool to appropriately set-up (and often narrow) the frame for analysis. Going the other direction, the analytic time unit is a way to aggregate up from the maximum granularity at which the data is collected. In some cases, the analytic time unit may be the same as the unit at which the data is collected; however in many cases it is necessary to aggregate across multiple units of collected data to create a meaningful analytic unit. For example, Schneider and Pea (2013) collected eye-tracking data from collaborating students at a rate of 30 data points per second, but the analytic time unit they used to establish students' joint visual attention was 4 seconds (based on prior research suggesting a time of $\sim 2$ seconds for a person to focus on an object mentioned by someone else). In other words, the criterion for joint attention was that students looked at the same area of the screen within +/- 2 seconds of each other, not the same 1/30 of a second. Analytic time units can be created to be exclusive (e.g. aggregate over 0-4 sec then 4-8 sec) or overlapping (also referred to as "sliding" e.g. aggregate over 0-4 sec then 1-5 sec) and need not always be of a constant length. Importantly, while such aggregation is often necessary, there is not always a clear theoretical basis on which to make such decisions. This creates a dilemma for the researcher who must find a rational, rather than arbitrary, way to make the determination. Importantly, the choice of time window can have dramatic effects on the patterns found in the data (Dyke, Kumar, Ai, \& Rosé, 2012); thus in some cases an empirical exploration of the robustness of analysis / interpretation with respect to the choice of analytic time unit is warranted.

Beyond establishing the grain size of the time window and analytic time unit, we need to translate theoretical conceptions of temporality into appropriate analytic methods. For example, if a theoretical 
(2017). Time for change: Why Learning analytics needs temporal analysis. Journal of Learning Analytics, 4(3), 7-17. http://dx.doi.org/10.18608/jla.2017.43.2

model of a problem-solving discussion relates to the sequencing of turn taking and its dynamics (e.g. cycles of initiation-response-feedback), then methods which model the likelihood with which one kind of action follows another can be used (e.g. lag sequence analysis, see, Chen, Resendes, Chai, \& Hong, 2017; Faraone \& Dorfman, 1987; Putnam, 1983). Another theoretical model might posit that problemsolving moves from a divergent to a convergent phase with certain kinds of actions more likely in one phase than the other (e.g. the divergent phase will have more new ideas introduced). In this case, the discussion could be modeled as a Markov process, specifying the probability of actions associated with each state and the probably of transitions between the states. Both of these analyses are best suited to short recurring sequences and analysis of event transitions (Reimann, 2009). In contrast optimal sequence matching (OSM) provides no information about transitions between events but can be used to identify the presence of similar sequences across data (including repeating sub-sequences within a larger series). Deciding which analysis to use and how to set up the models is thus intimately tied to, and should be guided by, underlying theoretical conceptions of the phenomenon being studied (Wise \& Shaffer, 2015). Moreover, additional work is required to support researchers in combining these analytic approaches to investigate these different temporal features and their relationship to learning.

\subsection{Pragmatic Challenges}

Some of these methodological challenges described above relate to pragmatic issues regarding to the collection, analysis, and manipulation (in end-user tools) of data at the appropriate scale. For example, while data collection decisions should be driven by well conceptualised temporal constructs, they may also be shaped (and limited) by practical concerns regarding available data formats, transfer speeds, and storage. Technological developments are likely to make significant inroads into this issue as well as the development of new learning tools in which data collection is taken as a foundational concern in design.

A different kind of pragmatic challenge is related to questions of impact and implementation that make temporal analytics actionable. Temporal analytics work is often laborious and lengthy to conduct and complex to interpret, creating barriers for classroom or online learning use. To make temporal analytics impactful and actionable we must develop techniques and systems to gather and analyse data in live learning contexts. Such work must also consider how insights from temporal analyses can be concretely used to inform the activities of teachers and students in the moment. For example, in what contexts and in what ways should an instructor act on information regarding learning sequences?

\section{A TRAJECTORY OF TEMPORAL CONSIDERATIONS IN LEARNING ANALYTICS}

Attention to these temporal concerns is growing in the learning analytics community and across the learning sciences and behavioural sciences more broadly. Indeed, due to the number of submissions received for this special section, we have decided to schedule it across two issues, the second of which will appear in early 2018. This double special section builds on a series of conference workshops over 
(2017). Time for change: Why Learning analytics needs temporal analysis. Journal of Learning Analytics, 4(3), 7-17. http://dx.doi.org/10.18608/jla.2017.43.2

which evolving perspectives on temporality can be seen. The First International Workshop on Temporality in Learning Data was held at the 2009 Technology-Enhanced Learning (TEL) Alpine Rendezvous in Germany and began an exploration of methods for characterizing and analyzing data related to collaboration and group learning over time. At the second workshop, held in 2010 at the International Conference of the Learning Sciences (ICLS) in Chicago, the focus shifted to the issues of how to analyze data streams collected across different time spans and at different levels of granularity (Cheng et al., 2010). In 2013, at the TEL Alpine Rendez-vous in France, initial efforts were made to look across different analytic approaches to map dimensions of temporal analyses in learning. The last two workshops have placed temporal considerations squarely into the realm of analytics, taking place at the International Learning Analytics and Knowledge Conference (LAK) in New York and Edinburgh. The 2015 workshop extended efforts to deepen understanding of distinct analytical approaches to studying temporality and the particular kinds of insight they can produce (Knight, Wise, Chen, \& Cheng. 2015). The 2016 workshop considered questions relating to the use of temporal analytics in educational practice, (Chen, Wise, Knight, \& Cheng, 2016).

Bringing together scholars from the learning sciences, learning analytics and technology-enhanced learning research communities, the workshops have increasingly foregrounded the importance of the temporal conceptualization of data, choosing appropriately among the wide variety of temporal analytic approaches possible, and developing research with early consideration of how to action analytics for practical impact in learning contexts. Critically, these challenges are interdisciplinary in nature, requiring input from experts in theory, methods and pedagogy. Thus, these concerns are an area where learning analytics and the learning sciences have clear synergistic potential and can engage in productive dialogue.

\section{PART ONE OF THE SPECIAL SECTION}

The temporal nature of learning cuts across a variety of data sources, learning constructs, and analysis technique with potential for impact on learning and learning contexts. Indeed, this diversity is reflected by the papers in this first part of the special section, which cover a broad range of data-types, learning constructs and analyses. Collectively, this work demonstrates the growing opportunities for collection and analysis of temporal data.

\subsection{Data Types}

Four of the papers in the first part of the special section provide analyses of discourse data that arise from a number of kinds of learning tools used in a variety of different contexts over a variety of time periods. Both Lee and Tan (this issue) and Demmans Epp, Phirangee and Hewitt, (this issue) analyse data from asynchronous computer supported collaborative learning environments (Knowledge Forum and Pepper respectively), specifically analysing 'notes' that student made in these environments distributed over a period of multiple weeks as part of university coursework. In contrast, Siebert-Evenstone et al. 
(2017). Time for change: Why Learning analytics needs temporal analysis. Journal of Learning Analytics, 4(3), 7-17. http://dx.doi.org/10.18608/jla.2017.43.2

(this issue) looked at synchronous data from an online virtual Internship environment in which students engage in mediated chat regarding a set of tasks that they must collaborate on. Lund, Quignard and Shaffer, (this issue) also analyse a synchronous conversation, but one which took place face-to-face between two students at lunchtime regarding feedback given to one of them by a teacher (the corpus was previously transcribed and annotated). Finally, Andrade, Danish and Maltese (this issue) focused on hand gesture data. This work took a more traditional lab based approach, capturing data from third and fourth graders as they spent a half hour completing of a set of tasks within the "Embodied Simulation of Population Dynamics" tool, a system designed to support students in embodied learning about complex systems. Beyond the specific characteristics of each data set, we note that Lund, et al.'s work makes use of the openly available data set "Meal Conversations Amongst Students". The sharing of data as a step towards supporting analytic reproducibility and triangulation across analyses is something this journal strongly supports (see Knight, Wise, Ochoa, and Hershkovitz, 2017).

\subsection{Temporal Learning Constructs \& Analysis Approaches}

A range of foci can also be seen in the temporal constructs being probed in each paper and the analyses to which they give rise. For example, Lee and Tan draw on 'idea promisingness' - a construct receiving increasing interest in the learning sciences. In this study, the authors first identify ideas that a knowledge building community found to be promising, as ones whose betweenness centrality (BC) in an ideas network shows a gradual, rather than sharp, drop after peak BC when it is introduced. They then examine the "mobility" or "drift" of these ideas over time across a two-dimensional BC / DC (degree centrality) space. These novel analyses both conceptualize temporality in terms of flow of elapsed time; in particular the rate of change of centrality of an idea over time. In the first analysis temporal properties are used simply as a tool for identification of promising ideas, while the second analysis results in temporal knowledge claims about the change in the importance of these ideas to the community over time. In the case of Demmans Epp, et al., temporality was also conceived as flow over time, but here the focus was on discussion participation activities and pronoun use (e.g. "I" "we" "you) as indicators of discussion health. Graphical representations and week by week statistical comparisons in combination with qualitative analysis of the actual student contributions were used to document differences between peer- and instructor-facilitated courses in the amount and consistency of interaction as well as pronoun use.

In contrast, Andrade, et al., study temporality through the lens of sequence, rather than flow. They also build their analyses specifically based on the use of a bespoke, theoretically designed, learning tool. In this case, the strongly theorised account of hand movements as an important embodied component of learning that is embedded in the tool design also guides the data generation. This allows the researchers to craft their analysis approach based on expectations of specific kinds of information the tool will provide and the ways these offer meaningful insight into the learning processes at play. Specifically, the authors investigate the elicitation of gesture as a means to support student learning of quantitative patterns of population dynamics (notably, a temporal phenomenon itself), suggesting that such gestures 
(2017). Time for change: Why Learning analytics needs temporal analysis. Journal of Learning Analytics, 4(3), 7-17. http://dx.doi.org/10.18608/jla.2017.43.2

can become objects to think with that are used to support explanation and collaboration. In their analysis they conceptualize temporality as an order of events, using optimal sequence matching (OSM) to compare the sequence of hand movements elicited by the learning tool with those enacted by the student (that are temporally shifted as the student takes time to respond to what the systems asks of them).

Siebert-Everstone et al., explore how temporal proximity is important in establishing connections across utterances between the kinds of talk used in an online chat. They do this to investigate the use of language indicative of taking on the identity of a particular profession. Specifically, their work focuses on the construction of a meaningful analytic time unit, referred to in their work as a "stanza". Here the stanza is conceptualised as a "breaking up" of a conversation into segments; of course, as we highlight above, this segmentation can also be thought of in terms of aggregating individual turns of talk. In their work they construct the analytic time unit as four utterances (based on an initial qualitative analysis of the data). References to key elements of engineering design are connected when they fall within the same unit, producing a quite different network of connections than if this was done across the whole conversation. While temporal properties are critical in producing the network, it is important to note that this technique does not explicitly lead to knowledge claims about how connections between ideas change over time. However, the use of this analytic approach led to several follow-up analyses in which knowledge claims were explicitly temporal (e.g. that discussion of collaboration occurs at the start and end of the chat only, the way one student's contributions followed and built on another's).

Lund, et al., were interested in drawing associations between micro-scale linguistic and discourse elements (e.g. elongated voices, disagreement) and macro-scale phases of emotional colouring (e.g. fear and pride). Examining the lunchtime talk of two students around teacher feedback, they also implemented the moving stanza approach to creating connections across utterances (this time using a 20 line analytic unit) of the micro-scale elements and then compared these across macro-scale phases (their larger time windows) using both the interactive visualizations and network graphs of element connections. In addition, these quantitative representations were used to identify sections of the transcript for qualitative analysis. Their work shows the ways in which multiple kinds of analytics approaches can be used complementarily to identify temporal features of discourse and to draw connection across constructs that exist at different timescales.

Overall, in conducting their analyses, the five papers draw on a range of approaches to validation, either using multiple quantitative techniques to generate insight about the target constructs or uniting statistical analysis with deep qualitative examinations of the data. Such efforts towards triangulation are important for validating results and offering robust interpretations of the data with which to inform practice. 
(2017). Time for change: Why Learning analytics needs temporal analysis. Journal of Learning Analytics, 4(3), 7-17. http://dx.doi.org/10.18608/jla.2017.43.2

\subsection{Potential Impact}

Across the papers, potential implications for supporting learning are highlighted, with further work required to explore and develop this impact. As Andrade, et al., note, fine-grained multimodal data including of course, discourse - has potential to transform learning not only through personalization and prediction, but also through strengthening our understanding of learning over time, and building our conceptual grounding for how to impact this. Perhaps most clearly, Demmans Epp, et al., draw out some practical pointers. They first show change in language over time as it relates to a target construct (feeling supported). Importantly, they then point to the potential for intervention by showing differences in student behaviours and language use that were associated with the facilitation method instructors adopted for their course. In a similar vein, Lee and Tan's work flags significant potential to analyse and support the development of promising ideas in online platforms. Important concerns are also raised at a methodological level, for example with all of the papers discussing concerns regarding aggregation of data and the appropriate unit or segmentation of data for temporal analyses. Lund, et al., and Siebert-Everstone et al., in particular focus on the size of the analytic time unit, showing how particular methods can provide insight into interactions that alternative approaches do not. This work has potential for providing real-time group and individual performance information that can drive instructor decision-making by drawing out relationships between phases of a learning activity, and the nature of the language being used in those phases.

\section{FUTURE DIRECTIONS}

Each of the papers in our double special section on temporal considerations in learning analytics makes an important contribution to furthering work in the space, but much work remains to be done to address the conceptual, methodological and pragmatic challenges described above. Such work may best be pursued both generally across the field, and specifically within sub-fields as they tackle temporality and its conceptualization in specific contexts. For example, within the sub-field of self-regulated learning $(S R L)$, a group of researchers has made substantial progress in both conceptualizing and operationalizing temporal constructs (Molenaar \& Järvelä). The high representation of papers in this special section looking at collaborative discourse suggests that this may be another sub-field ripe for collective conceptualization.

Nevertheless, across this work, and the field at large, some core concerns remain. In the main editorial for this issue of the Journal of Learning Analytics the editors flag the potential of convergence across the various research communities pursuing learning analytics work, but also highlight the challenges that reviewers face in evaluating interdisciplinary work. Such challenges become amplified in the context of temporal work which necessarily introduces concepts and/or methodologies with which many in the field may not be familiar. For example, as a field that seeks to draw together computational approaches with strongly theorised learning concerns, we must navigate both established and emerging theory and methodological innovation. The application of established temporal methods from other disciplines can 
(2017). Time for change: Why Learning analytics needs temporal analysis. Journal of Learning Analytics, 4(3), 7-17. http://dx.doi.org/10.18608/jla.2017.43.2

provide fruitful material to support the development of the field. Equally, we must not forget that learning analytics is about learning (Gašević, Dawson \& Siemens, 2015). This draws attention to the central pragmatic challenge of making sure to always ask "what impact will this have on learning?".

As a field we must navigate the space to ensure that we can effectively draw on a range of methods and datasets to provide illustrative examples of the application of analytics, even where learning outcome data may not be directly available. Indeed, as Lund, et al., illustrate, analysis of data that is not itself "pedagogic" in nature can still inform our understanding of learning contexts. We note, though, that these connections must be made clear and explicit, with an obvious onward trajectory to impact on learning. We hope that the "Notes for Practice" section of each article (a new innovation in the Journal of Learning Analytics, see Knight, et al., 2017) provides one means through which to foreground potential implications for practical learning contexts.

Finally, we highlight that there is need for independent advances against each of the challenges conceptual, methodological, and pragmatic - alongside research that seeks to integrate approaches to these challenges. This research may be conducted independently - with each paper addressing a particular challenge - or in an integrated way, with papers that seek to address the full learning analytics lifecycle. Either way, as a community we must work to develop research such that methodological advances by team $A$ can lead to improved temporal conceptualisation by team $B$ and integrated pragmatic advances by team $C$, such that we each build on each other's work. Silos of contributions are unlikely to achieve the impact we need. The time is ripe for the development of learning analytics that take full advantage of the temporal features of learning data, and in doing so help us enrich our understanding and ability to support learning as a process of change over time.

\section{ACKNOWLEDGEMENTS}

We would like to acknowledge the contributions of Britte Haugan Cheng, Inge Molenaar, Ming Ming Chiu, Vanessa Svihla, Vanessa Peters, Katerina Zourou, Dan Suthers, Murat Cakir, Manu Kapur, and Timothy Zimmerman who were all instrumental in the development of the temporality workshop series that laid the foundation for this special issue. In particular Britte Cheng took on a leadership role in each of the temporality workshops and provided invaluable input and support for the theoretical and methodological progress that they facilitated.

\section{REFERENCES}

Akhras, F. N., \& Self, J. A. (1999). Modeling the process, not the product, of learning. In S. P. Lajoie P. \& S. J. Derry (Eds.), Computers as cognitive tools (Vol. 2, pp. 3-28). Hillsdale, N.J.,: Lawrence Erlbaum Associates.

Barbera, E., Gros, B., \& Kirschner, P. (2014). Paradox of time in research on educational technology. Time \& Society. https://doi.org/10.1177/0961463X14522178 
(2017). Time for change: Why Learning analytics needs temporal analysis. Journal of Learning Analytics, 4(3), 7-17. http://dx.doi.org/10.18608/jla.2017.43.2

Biswas, G., Jeong, H., Kinnebrew, J. S., Sulcer, B., \& Roscoe, R. (2010). Measuring self-regulated learning skills through social interactions in a teachable agent environment. Research and Practice in Technology Enhanced Learning, 5(02), 123-152. https://doi.org/10.1142/s1793206810000839

Blikstein, P. (2011). Using learning analytics to assess students' behavior in open-ended programming tasks. In Proceedings of the 1st international Conference on Learning Analytics and Knowledge (pp. 110-116). Edmonton, AB: ACM. https://doi.org/10.1145/2090116.2090132

Cepeda, N. J., Pashler, H., Vul, E., Wixted, J. T., \& Rohrer, D. (2006). Distributed practice in verbal recall tasks: A review and quantitative synthesis. Psychological bulletin, 132(3), 354. https://doi.org/10.1037/0033-2909.132.3.354

Chen, B., Resendes, M., Chai, C. S., \& Hong, H.-Y. (2017). Two tales of time: uncovering the significance of sequential patterns among contribution types in knowledge-building discourse. Interactive Learning Environments, 25(2), 162-175. https://doi.org/10.1080/10494820.2016.1276081

Chen, B., Wise, A. F., Knight, S., \& Cheng, B. H. (2016). It's About Time: Putting Temporal Analytics into Practice: The 5th International Workshop on Temporality in Learning Data (pp. 488-489). In Proceedings of at the 6th ACM Learning Analytics and Knowledge Conference, Edinburgh, UK: ACM. https://doi.org/10.1145/2883851.2883865

Cheng, B. H., Molenaar, I., Chiu, M. M., Svihla, V., Wise, A., Peters, V., \& Zourou, K. (2010). It's about time: purpose, methods, and challenges of temporal analyses of multiple data streams. In Proceedings of the 9th International Conference of the Learning Sciences-Volume 2 (pp. 501502). International Society of the Learning Sciences. https://dl.acm.org/citation.cfm?id=1854770

Dyke, G., Kumar, R., Ai, H., \& Rosé, C. P. (2012). Challenging assumptions: Using sliding window visualizations to reveal time-based irregularities in CSCL processes. Proceedings of the International Conference of the Learning Sciences (pp. 363370). Sydney, Australia: ICLS. https://pdfs.semanticscholar.org/c88d/1081e9414c23046cacda127528832f0728b8.pdf

Faraone, S. V., \& Dorfman, D. D. (1987). Lag sequential analysis: Robust statistical methods. Psychological Bulletin, 101(2), 312-323. https://doi.org/10.1037/0033-2909.101.2.312

Gašević, D., Dawson, S., \& Siemens, G. (2015). Let's not forget: Learning analytics are about learning. TechTrends, 59(1), 6471. https://doi.org/10.1007/s11528-014-0822-x

Gunawardena, C. N., Lowe, C. A., \& Anderson, T. (1997). Analysis of a global online debate and the development of an interaction analysis model for examining social construction of knowledge in computer conferencing. Journal of educational computing research, 17(4), 397-431. https://doi.org/10.2190/7mqv-x9uj-c7q3-nrag

Halatchliyski, I., Hecking, T., Goehnert, T., \& Hoppe, H. U. (2014). Analyzing the main paths of knowledge evolution and contributor roles in an open learning community. Journal of Learning Analytics, 1(2), 72-93. https://doi.org/10.18608/jla.2014.12.5

Haythornthwaite, C., \& Gruzd, A. (2012). Exploring patterns and configurations in networked learning texts. In 45th Hawaii International Conference on System Science (HICSS), (pp. 3358-3367). Maui, HI: IEEE. https://doi.org/10.1109/hicss.2012.268 
(2017). Time for change: Why Learning analytics needs temporal analysis. Journal of Learning Analytics, 4(3), 7-17. http://dx.doi.org/10.18608/jla.2017.43.2

Kapur, M., Voiklis, J., \& Kinzer, C. K. (2008). Sensitivities to early exchange in synchronous computersupported collaborative learning (CSCL) groups. Computers \& Education, 51(1), 54-66. https://doi.org/10.3115/1599600.1599663

Knight, S., Wise, A. F., Chen, B., \& Cheng, B. H. (2015). It's About Time: 4th International Workshop on Temporal Analyses of Learning Data (pp. 388-389). In the Proceedings of the 5th International Learning Analytics \& Knowledge Conference (LAK15): Scaling Up: Big Data to Big Impact, Poughkeepsie, NY, USA: ACM. https://doi.org/10.1145/2723576.2723638

Knight, S., Wise, A. F., Ochoa, X., \& Hershkovitz, A. (2017). Learning Analytics: Looking to the Future. Journal of Learning Analytics, 4(2), 1-5. https://doi.org/10.18608/jla.2017.42.1

Littleton, K. (1999). Productivity through interaction. In K. Littleton \& P. Light (Eds.), Learning with computers: Analysing productive interaction (pp. 179-194). London, UK: Routledge.

Mercer, N. (2008). The seeds of time: why classroom dialogue needs a temporal analysis. Journal of the Learning Sciences, 17(1), 33-59. https://doi.org/10.1080/10508400701793182

Mercer, N., \& Littleton, K. (2007). Dialogue and the development of children's thinking: A Sociocultural Approach (New edition). Oxon: Routledge.

Molenaar, I., \& Järvelä, S. (2014). Sequential and temporal characteristics of self and socially regulated learning. Metacognition and Learning, 9(2), 75-85. https://doi.org/10.1007/s11409-014-9114-2

Putnam, L. L. (1983). Small group work climates A lag-sequential analysis of group interaction. Small Group Research, 14(4), 465-494. https://doi.org/10.1177/104649648301400405

Reimann, P. (2009). Time is precious: Variable-and event-centred approaches to process analysis in CSCL research. International Journal of Computer-Supported Collaborative Learning, 4(3), 239-257. https://doi.org/10.1007/s11412-009-9070-z

Schneider, B., \& Pea, R. (2013). Real-time mutual gaze perception enhances collaborative learning and collaboration quality. International Journal of Computer-supported collaborative learning, 8(4), 375-397. https://doi.org/10.1007/s11412-013-9181-4

Wise, A. F., \& Chiu, M. M. (2011). Analyzing temporal patterns of knowledge construction in a role-based online discussion. International Journal of Computer-Supported Collaborative Learning, 6(3), 445-470. https://doi.org/10.1007/s11412-011-9120-1

Wise, A. F., Perera, N., Hsiao, Y., Speer, J., \& Marbouti, F. (2012). Microanalytic case studies of individual participation patterns in an asynchronous online discussion in an undergraduate blended course. The Internet and Higher Education, 15(2), 108-117.

Wise, A. F. \& Shaffer, D. W. (2015). Why theory matters more than ever in the age of big data. Journal of Learning Analytics (Special Section on Learning Analytics and Learning Theory), 2(2), 5-13. https://doi.org/10.18608/jla.2015.22.2 